\title{
Asteroseismic analysis of eight solar-like oscillating evolved stars in the open cluster NGC 6811
}

\author{
Zeynep Çelik Orhan ${ }^{1 \star}$ \\ ${ }^{1}$ Department of Astronomy and Space Sciences, Science Faculty, Ege University, 35100, Bornova, İzmir, Turkey
}

Accepted XXX. Received YYY; in original form ZZZ

\begin{abstract}
The Kepler space telescope has provided exquisite data to perform asteroseismic analysis on evolved star ensembles. Studying star clusters offers significant insight into stellar evolution and structure, due to having a large number of stars with essentially the same age, distance, and chemical composition. This study analysed eight solar-like oscillating evolved stars that are members of the open cluster NGC 6811 and modelled them for the first time. The fundamental stellar parameters are obtained from the interior model using observational asteroseismic and non-asteroseismic constraints. The stellar interior models are constructed using the MESA evolution code. The mass-loss method is included in the interior models of the stars. The stellar masses and radii ranges of the stars are 2.23-2.40 $M_{\odot}$ and 8.47-12.38 $R_{\odot}$, respectively. Typical uncertainties for the mass and radius are $\sim 0.11 M_{\odot}$ and $\sim 0.09 R_{\odot}$, respectively. The model masses and radii are compared with masses and radii obtained from asteroseismic and non-asteroseismic methods (scaling relations and classic methods). The stellar ages fell in the range between 0.71 and 0.82 Gyr, with a typical uncertainty of $\sim 18$ per cent. The model ages of the star calculated in this study are compatible with those reported in the literature for NGC 6811.
\end{abstract}

Key words: stars: fundamental parameters - stars: oscillations - stars: interiors - stars: evolution.

\section{INTRODUCTION}

Asteroseismology is a prominent observation method that offers the potential to probe stellar interiors and test interior models. The best-fitting model and observed individual oscillation frequencies can give precise estimates of stellar parameters, but these parameters are model-dependent. For example, the helium abundance is an uncertain model input parameter. It is difficult to obtain the helium abundance from spectra, thus the approximate values given by the Galactic element enrichment law is widely accepted in theoretical models. Recent studies showed that analysing acoustic glitches in oscillation frequencies could constrain the surface helium abundances $(Y)$, but the analysis requires very high-quality observed asteroseismic data (Verma et al. 2014, 2017). In addition, evolved stars are expected to lose mass on the red giant branch (RGB), but the amount of mass loss is an important unsolved problem. Therefore, particular attention has been devoted to the three open clusters in the Kepler field (NGC 6791, NGC 6811, and NGC 6819; Stello et al. 2010; Basu et al. 2011; Hekker et al. 2011; Stello et al. 2011a, 2011b; Miglio et al. 2012) due to the well-known fact that cluster stars share the same age, distance, and chemical composition, thereby allowing more stringent investigations into stellar evolution theory. In this study, the interior models for eight evolved

\footnotetext{
* E-mail: zeynep.celik@ege.edu.tr
}

stars in the NGC 6811 are constructed using the MESA evolution code. By combining asteroseismic and non-asteroseismic observational constraints, fundamental stellar parameters are obtained very precisely from interior models.

The stellar mass plays the most important role in aiding further understanding stellar evolution and structure, therefore it needs to be determined very precisely. However, determining the stellar masses from observations is difficult, except for eclipsing binary stars. Currently, the masses and radii of solar-like oscillating stars can be directly determined using observational data. When the large separation oscillation frequencies $(\Delta \nu)$, frequency of the maximum amplitude $\left(\nu_{\max }\right)$, and effective temperature $\left(T_{\text {eff }}\right)$ are known, the masses and radii of solar-like oscillating stars are calculated from conventional scaling relations. These conventional scaling relations (Kjeldsen and Bedding 1995) assume that the solar-like oscillating stars have similar internal structure conditions. This assumption causes systematic errors in the $M$ and $R$ calculations. A large number of studies have proposed new methods of reducing these systematic errors (Bellinger 2019, Sharma et al. 2016, Yıldız, Çelik Orhan \& Kayhan 2016; hereafter Paper III, White et al. 2011; Mosser et al. 2013; Guggenberger et al. 2016, Yıldız et al. 2014a; hereafter Paper I; Yıldız et al. 2015; hereafter Paper II).

On the other hand, with the classical method, $M$ and $R$ are determined using non-asteroseismic observation parameters. This method involves using distance to calculate stel- 
lar $M$ and $R$. Thanks to the Gaia space telescope, the distances of Kepler target stars are determined very precisely. The distances values of eight evolved stars were taken from Gaia database. In this method, the star's bolometric correction values from the MESA Isochrones \& Stellar Tracks (MIST) bolometric tables are used. From here, the luminosity of the star is calculated. The stellar radius is determined from the luminosity. The mass is then calculated using the observational logg. In this way, the $M$ and $R$ of the stars are determined using Gaia DR3 parallax, observed effective temperature and observed surface gravity and MIST bolometric correction tables (Choi et al. 2016; Dotter 2016).

Another method is based on calculating the mass and radius by fitting stellar internal structure models with the nonasteroseismic observation parameters of the star. However, the uncertainties in $M$ and $R$ determined by this method are often large. Therefore, in this study, non-asteroseismic ( $[\mathrm{Fe} / \mathrm{H}]$, distance, $\log g$, and $\left.T_{\text {eff }}\right)$ and the standard asteroseismic observation parameters $\left(\Delta \nu, \nu_{\max }\right)$ and, two reference frequencies $\left(\nu_{\min 0}\right.$ and $\left.\nu_{\min 1}\right)$ are used while fitting the internal structure model. The quantities $\left(\nu_{\min 0}\right.$ and $\left.\nu_{\min 1}\right)$ are determined from a $\Delta \nu$ versus $\nu$ graph and are the references frequencies corresponding to the highest and lowest values of the pairwise $\Delta \nu$, respectively (see figure 3 of Paper I). In this way, the best-fitting model that represents the star can be constructed.

In this study, eight solar-like evolved stars in the open cluster NGC 6811 were selected and analysed them using the MESA evolution code. All stellar models have included the mass-loss method. This method was necessary to calibrate the stellar ages of the cluster member stars. Model $M$ and $R$ values obtained by different methods were compared in detail. These different methods include conventional scaling relations, modified scaling relations (in Paper III), Bellinger methods (Bellinger 2019), and classic methods. The model oscillation frequencies are calculated using the ADIPLS package (Christensen-Dalsgaard 2008). The reference frequencies $\left(\nu_{\text {mins }}\right)$, other asteroseismic $\left(\nu_{\max }\right.$ and $\left.\Delta \nu\right)$, and non-asteroseismic parameters (effective temperature, surface gravity, and metallicity) are used to calibrate the interior models. Thus, $M, R$, luminosity $(L)$, gravity $(\log g)$ and age $(t)$ of the stars are obtained very precisely from the interior models using observed asteroseismic and non-asteroseismic parameters.

This work is organised as follows. Section 2 presents the observational properties of eight solar-like oscillating evolved stars of NGC 6811. The properties of the MESA evolution code, model calculation method, and the different methods used to calculate mass and radius are described in Section 3. In Section 4, the model and scaling relations results obtained for these stars are compared. Finally, Section 5 presents the results.

\section{ASTEROSEISMIC AND NON-ASTEROSEISMIC PROPERTIES OF THE EIGHT EVOLVED STARS IN NGC 6811}

The period spacing carries information about the evolutionary state of an evolved star. The range of observed period spacing of the stars is 100-190 s (Arentoft et al. 2017). As a result, when the observed period spacing values analysed us-

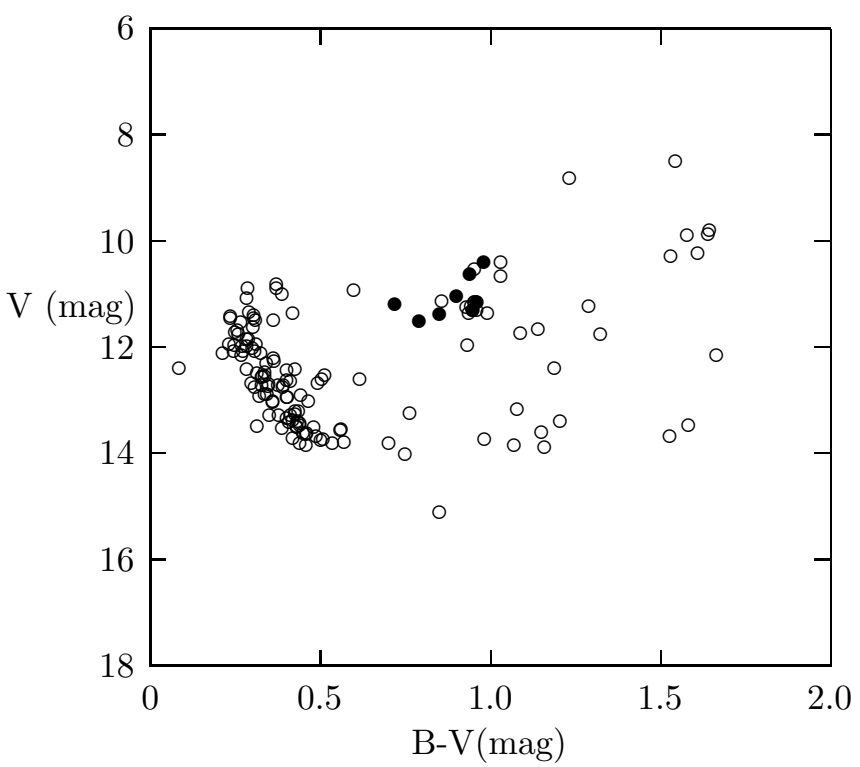

Figure 1. The B-V versus $\mathrm{V}$ diagram is plotted for eight solar-like oscillating evolved stars (filled circles) and other cluster member stars (circles) in NGC 6811.

ing data from Bedding et al. (2011, see fig.3), it shows that the evolved stars are red-clump (RC) (Pinsonneault et al. 2018). According to the WEBDA database, the open cluster NGC 6811 is an intermediate-age $(\log ($ age $)=8.799)$, and slightly reddened $(E(B-V)=0.160 \mathrm{mag})$ cluster that is located at a distance of 1215 pc. In Fig. 1, the eight evolved stars are plotted in a color-magnitude diagram. While the filled circles in Fig. 1 represent the eight solar-like oscillating evolved stars modelled in this study, the circles represent the NGC 6811 cluster stars taken from $W E B D A$ database. Their B-V and $\mathrm{V}$ values were taken from $S I M B A D$ database.

The solar-like oscillations for the eight evolved stars in NGC 6811 have been detected by Arentoft et al. (2017). Table 1 lists the observed stellar asteroseismic and nonasteroseismic properties. Their light curves are observed by the Kepler space telescope. They show mixed mode oscillations on the observed frequencies. $\Delta \nu, \nu_{\max }$, and mean small separation between oscillation frequencies $\left(\delta \nu_{02}\right)$ are derived by Arentoft et al. (2017). The observed and model $\Delta \nu$ fit are determined from $\Delta \nu$ versus $\nu$ graphs (see Fig. 2). Reference frequencies are also obtained from the $\Delta \nu$ versus $\nu$ graphs.

$\nu_{\min 0}$ and $\nu_{\min 1}$ have been determined from their $\Delta \nu$ versus $\nu$ graph for eight evolved stars (see Fig. 2). As seen in Figure 2, decreases in observation and model frequencies are seen in the $\Delta \nu$ versus $\nu$ graph. The frequencies corresponding to the high and low frequencies from these drops are named as $\nu_{\min 1}$ and $\nu_{\min 0}$, respectively. The value of $\nu_{\min 2}$ for these stars could not be determined from observed radial oscillation frequencies. The method suggested in Paper I was used when determining these frequencies, which occur due to $\mathrm{He}$ II glitch. First, the frequency interval of the minimum in $\Delta \nu$ versus $\nu$ graph was determined. In this frequency interval, two lines were drawn from the neighboring intervals. The intersection of the two lines gives $\nu_{\text {min }}$ which are the reference frequencies. Uncertainties in reference frequencies are calculated from half of the observed large separation (Paper III). The uncertainties in $\nu_{\min 0}$ and $\nu_{\min 1}$ are listed in Table 1. 
Table 1. Observed properties of eight solar-like oscillating evolved stars. The listed asteroseismic and non-asteroseismic properties are parallax $(\pi)$ from Gaia database and magnitude $(\mathrm{G})$, effective temperature from spectra ( $\left.T_{\mathrm{es}}\right)$, logarithmic of surface gravity (logg), $[F e / H], \Delta \nu$, small separation $\left(\delta \nu_{02}\right), \nu_{\max }, \nu_{\min 0}$, and $\nu_{\min 1}$ respectively.

\begin{tabular}{lcccccccccc}
\hline KIC & $\pi$ & $\mathrm{G}$ & $T_{\mathrm{es}}$ & $\log g$ & {$[\mathrm{Fe} / \mathrm{H}]$} & $\Delta \nu$ & $\nu_{\max }$ & $\nu_{\min 0}$ & $\nu_{\min 1}$ \\
& $(\mathrm{mas})$ & $(\mathrm{mag})$ & $(\mathrm{K})$ & $(\mathrm{cgs})$ & $(\mathrm{dex})$ & $(\mu \mathrm{Hz})$ & $(\mu \mathrm{Hz})$ & $(\mu \mathrm{Hz})$ & $(\mu \mathrm{Hz})$ \\
& & & & & & & & & \\
& & & & & & & & \\
\hline 9409513 & $0.9866 \pm 0.02$ & $10.24 \pm 0.03$ & $4950 \pm 100$ & $2.76 \pm 0.02$ & $-0.050 \pm 0.050$ & $6.04 \pm 0.02$ & $69.8 \pm 1.0$ & $81.75 \pm 3.04$ & $61.90 \pm 3.04$ \\
9532903 & $0.8485 \pm 0.02$ & $10.91 \pm 0.03$ & $5055 \pm 100$ & $2.93 \pm 0.02$ & $-0.007 \pm 0.020$ & $7.55 \pm 0.04$ & $92.0 \pm 1.5$ & $105.86 \pm 3.78$ & $81.05 \pm 3.78$ \\
9534041 & $0.8516 \pm 0.02$ & $11.02 \pm 0.03$ & $5039 \pm 100$ & $2.99 \pm 0.02$ & $-0.052 \pm 0.050$ & $8.35 \pm 0.01$ & $103.8 \pm 1.0$ & $117.64 \pm 4.18$ & $87.08 \pm 4.18$ \\
9655101 & $0.8658 \pm 0.02$ & $10.97 \pm 0.03$ & $5062 \pm 100$ & $2.94 \pm 0.02$ & $-0.039 \pm 0.050$ & $7.88 \pm 0.02$ & $98.7 \pm 1.0$ & $103.50 \pm 3.94$ & $83.89 \pm 3.94$ \\
9655167 & $0.8652 \pm 0.02$ & $11.04 \pm 0.03$ & $5048 \pm 100$ & $2.93 \pm 0.02$ & $-0.006 \pm 0.020$ & $8.07 \pm 0.01$ & $99.4 \pm 2.1$ & $105.23 \pm 4.04$ & $88.99 \pm 4.04$ \\
9716090 & $0.8483 \pm 0.02$ & $11.09 \pm 0.03$ & $5013 \pm 100$ & $2.70 \pm 0.02$ & $-0.050 \pm 0.050$ & $8.54 \pm 0.02$ & $107.8 \pm 1.4$ & $119.78 \pm 4.27$ & $94.11 \pm 4.27$ \\
9716522 & $0.8124 \pm 0.02$ & $10.41 \pm 0.05$ & $4861 \pm 100$ & $2.64 \pm 0.03$ & $-0.030 \pm 0.020$ & $4.88 \pm 0.01$ & $53.7 \pm 1.0$ & $58.10 \pm 2.44$ & $48.21 \pm 2.44$ \\
9776739 & $0.8639 \pm 0.02$ & $10.90 \pm 0.05$ & $5118 \pm 100$ & $2.92 \pm 0.03$ & $0.010 \pm 0.020$ & $7.65 \pm 0.03$ & $92.9 \pm 1.0$ & $110.33 \pm 3.83$ & $82.29 \pm 3.83$ \\
\hline
\end{tabular}

Spectroscopic data of Kepler target NGC 6811 cluster member stars $\left(\log g,[\mathrm{Fe} / \mathrm{H}]\right.$, and $\left.T_{\text {eff }}\right)$ are taken from Hawkins et al. (2016). Distance is a crucial stellar parameter. Thanks to the Gaia space telescope, a large number of star distances are very precisely determined. Parallax $(\pi)$ and G magnitude are derived from the Gaia DR3 database. The effective temperature ranges between $4826 \mathrm{~K}$ (KIC 9716522) and $5027 \mathrm{~K}$ (KIC 9534041). The [Fe/H] ranges between -0.052 (KIC 9534041) and 0.010 dex (KIC 9776739).

\section{PROPERTIES OF INTERIOR MODELS OF THE STARS}

\subsection{Properties of MESA evolution code}

The stellar interior models are constructed using the MESA evolution code (version 15140, Paxton et al. 2011, 2013). Based on calibration of the solar model, the values $\mathrm{Y}, \mathrm{Z}$ and, convective parameters $(\alpha)$ are computed as $0.2745,0.0172$, and 1.8125 , respectively.

Convection is treated with a standard mixing-length theory (Böhm- Vitense 1958). The interior models do not consider microscopic diffusion effects. A small amount of convective overshoot as described by Herwig (2000) was allowed during both the main-sequence and red giant phases. The exponential convective overshoot parameter was fixed at $f_{0}=0.016$, based on the values used in the MIST isochrones (Choi et al. 2016). MESA offers the opacity tables of Iglesias \& Rogers $(1993,1996)$ and includes their OPAL opacity tables in the high-temperature region supplemented by low-temperature tables of Ferguson et al. (2005) with fixed metallicity as the a default option.

Stellar metallicity significantly affects pre-main sequence (pre-MS) phases as well as every other phase of the star. It is therefore important for us to start from this phase and evolve the star there. In this study, the pre-MS is included in the construction of stellar interior models. Nuclear reaction rates are taken from Angulo et al. (1999) and Caughlan \& Fowler (1988). Mass-loss can be included into MESA in a variety of built-in parametrizations. Reimers' mass-loss law is applied for the stellar evolution models. Atmospheric conditions significantly effect the model oscillation frequencies determined. The simple_photosphere option is selected in the MESA code for the star interior models. The theoretical oscillation frequencies are computed by the ADIPLS pulsation package (Christensen-Dalsgaard 2008). To correct the model frequencies for so-called "surface effects" due to incomplete modeling of the near-surface layers, the empirical prescription of Kjeldsen et al. (2008) is used.

\subsubsection{Modelling strategy and uncertainty calculation}

The model input parameters are initial $M, Y, Z$, and $\alpha$. When a stellar model is constructed, all available observational data for that particular star are used. $M$ is the most important parameter affecting stellar structure and evolution. Therefore, an initial mass value must be entered in the model calculations. This study uses three different methods to determine the initial mass value (see Sect.3.1). Since scaling relations are developed for MS stars, $M_{\pi}$ is taken as the first mass in the constructed star model. To calibrate the stellar interior models, the initial values of $M$ and $\alpha$ were changed until the observed asteroseismic and non-asteroseismic constraints were obtained in the models.

After mass, $Z$ is the parameter that mostly affects the stellar structure and evolution. Therefore, $Z$ is another important input parameter. In general, $Z$ is computed from the observed $[\mathrm{Fe} / \mathrm{H}]$ or overall metallicity $[\mathrm{M} / \mathrm{H}]$ reported in the literature. Then the total metallicity $(Z)$ from $[\mathrm{M} / \mathrm{H}]$ is computed using this method. $Z=10^{[\mathrm{Fe} / \mathrm{H}]} 0.0134$ is used for metallicity calculations of initial models. The metallicity in the present-day solar photosphere was determined as 0.0134 by Asplund et al. (2019). Therefore, in this study, the solar metallicity value of the eight evolved stars is taken as 0.0134 . Here, the lowest and the highest metal abundance are calculated as 0.0119 and 0.0370 , respectively. The average of the calculated metallicity of the stars is determined as 0.0126 and the initial $Y$ is set to as 0.2745 (solar value).

The model calibration procedure is the same as that of Çelik Orhan, Yıldız \& Kayhan (2021) for modelling of 15 evolved stars (see Section 3.2). The compatibility between models and the non-asteroseismic and asteroseismic observational data is examined. For this purpose $\chi_{\mathrm{spec}}^{2}$ values are calculated from Eq. 6 (see Sec. 3.3). While examining the harmony between the observed and model parameters, special attention was 
Table 2. List of model properties of evolved solar-like oscillating stars. $M_{\mathrm{mod}}, R_{\mathrm{mod}}, T_{\mathrm{mod}}, L_{\mathrm{mod}}, \log g_{\mathrm{mod}}, \alpha, t_{\mathrm{mod}}$, and $\chi_{\mathrm{spec}}^{2}$, respectively, stellar mass in $M_{\odot}$ unit, stellar radius in $R_{\odot}$ unit, effective temperature in $K$ unit, luminosity in $L_{\odot}$ unit, logarithm of surface gravity of the model and stellar age in Gyr unit.

\begin{tabular}{lcccccccc}
\hline KIC & $\begin{array}{c}M_{\text {mod }} \\
\left(M_{\odot}\right)\end{array}$ & $\begin{array}{c}R_{\text {mod }} \\
\left(R_{\odot}\right)\end{array}$ & $\begin{array}{c}T_{\text {mod }} \\
(\mathrm{K})\end{array}$ & $\begin{array}{c}L_{\text {mod }} \\
\left(L_{\odot}\right)\end{array}$ & $\begin{array}{c}\log g_{\text {mod }} \\
(\mathrm{cgs})\end{array}$ & $\alpha$ & $\begin{array}{c}t_{\text {mod }} \\
(\mathrm{Gyr})\end{array}$ & $\chi_{\mathrm{spec}}^{2}$ \\
\hline 9409513 & $2.40 \pm 0.10$ & $10.69 \pm 0.09$ & $4985 \pm 100$ & $55.21 \pm 3.41$ & $2.76 \pm 0.01$ & 2.07 & $0.71 \pm 0.18$ & 0.25 \\
9532903 & $2.27 \pm 0.11$ & $9.17 \pm 0.08$ & $4992 \pm 100$ & $42.48 \pm 2.07$ & $2.87 \pm 0.02$ & 1.55 & $0.79 \pm 0.17$ & 0.23 \\
9534041 & $2.38 \pm 0.10$ & $9.05 \pm 0.08$ & $5025 \pm 100$ & $53.37 \pm 2.34$ & $2.90 \pm 0.02$ & 1.95 & $0.72 \pm 0.18$ & 0.13 \\
9655101 & $2.35 \pm 0.11$ & $9.09 \pm 0.09$ & $5060 \pm 100$ & $51.00 \pm 2.92$ & $2.91 \pm 0.02$ & 1.95 & $0.73 \pm 0.18$ & 0.15 \\
9655167 & $2.25 \pm 0.11$ & $8.79 \pm 0.09$ & $5044 \pm 100$ & $45.60 \pm 2.90$ & $2.90 \pm 0.01$ & 1.80 & $0.82 \pm 0.18$ & 0.25 \\
9716090 & $2.23 \pm 0.12$ & $8.47 \pm 0.08$ & $4997 \pm 100$ & $37.18 \pm 2.45$ & $2.93 \pm 0.01$ & 1.60 & $0.75 \pm 0.18$ & 0.13 \\
9716522 & $2.35 \pm 0.12$ & $12.38 \pm 0.12$ & $4824 \pm 100$ & $83.41 \pm 4.80$ & $2.62 \pm 0.01$ & 1.95 & $0.77 \pm 0.16$ & 0.05 \\
9776739 & $2.34 \pm 0.14$ & $9.24 \pm 0.09$ & $5120 \pm 100$ & $49.18 \pm 3.74$ & $2.88 \pm 0.01$ & 1.75 & $0.75 \pm 0.16$ & 0.07 \\
\hline
\end{tabular}

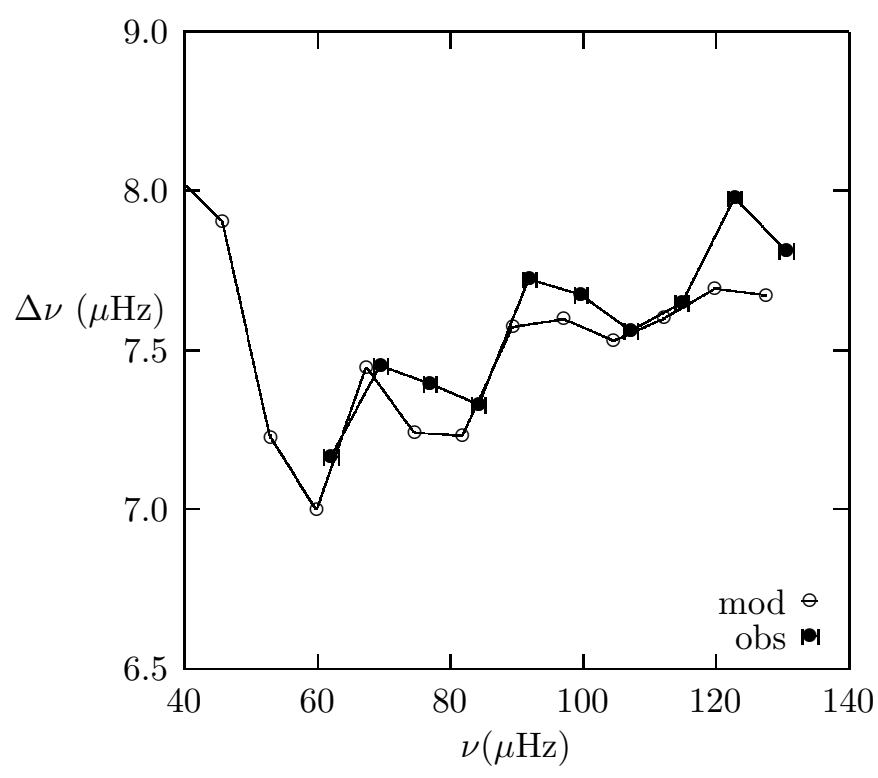

Figure 2. Plot of $\Delta \nu$ as a function of $\nu$ graph for model (MESA, circles) and observed frequencies (filled circles) of KIC 9776739.

paid to make sure that the $\chi_{\text {spec }}^{2}$ value is smaller than one. The obtained $\chi_{\text {spec }}^{2}$ values are listed in Table 2 .

In addition, reference frequencies and other seismic parameters were also examined, while seeking to determine the best model. In Fig 2, $\Delta \nu$ versus $\nu$ for KIC 9776739 is plotted for comparing observed and model oscillation frequencies as well as the reference frequencies. The observation frequencies of each star are close to those computed by the models. Special attention was paid to make sure that the model and observational $\Delta \nu$ values were the same. The fact that the patterns overlapped and the same on the $\Delta \nu$ versus $\nu$ graph is the most important feature of the model oscillation frequencies. Attention is paid to ensure that this value is below than one for the interior models. The obtained $\chi_{\text {seis }}^{2}$ are listed in Table 3 .

\subsubsection{Mass loss in interior models}

RGB mass loss is the content of most stellar evolution codes, and is generally obtained by simple relations of the stellar parameters. The most commonly used mass-loss method, the Reimers (1975) method, was used in this stellar interior models of these eight evolved stars in this study. However, using this method requires a reference point $(\eta)$. Assuming a Reimers mass-loss law, model comparisons suggest $\eta$ in the range 0-0.1 with a strong preference for no mass loss at all.

Red-clump (RC) stars are the only ones for which classical mass-loss formulas (Reimers 1975, Renzini \& Fusi Pecci 1988) can produce an appreciable mass loss. There are eight $\mathrm{RC}$ stars in this study. The stellar interior models of RC stars are constructed using Reimers (1975) mass-loss methods $(\eta=0.1)$. The total mass loss in all the internal structure models constructed is $0.01 M_{\odot}$.

\subsubsection{The uncertainties calculations}

To quantify the differences between the model and observational data obtained for each star, a normalised $\chi^{2}$ was calculated for asteroseismic and non-asteroseismic constraints:

$\chi_{\text {seis }}^{2}=\frac{1}{N_{\mathrm{f}}} \sum_{i=1}^{n}\left(\frac{\nu_{\mathrm{i}, \mathrm{obs}}-\nu_{\mathrm{i}, \mathrm{mod}}}{\sigma_{\mathrm{i}, \mathrm{obs}}}\right)^{2}$

and

$\chi_{\mathrm{spec}}^{2}=\frac{1}{N_{\mathrm{s}}} \sum_{i=1}^{n}\left(\frac{P_{\mathrm{i}, \mathrm{obs}}-P_{\mathrm{i}, \mathrm{mod}}}{\sigma_{\mathrm{i}, \mathrm{obs}}}\right)^{2}$

where $\nu_{\mathrm{i}, \text { obs }}$ and, $\nu_{\mathrm{i}, \bmod }$ are the observed and model oscillation frequencies, respectively. $N_{\mathrm{f}}$ is the total number of the modes for $l=0, \sigma_{\mathrm{i}, \mathrm{obs}}$ is the uncertainty of the observed frequencies. $\nu_{\max }, \Delta \nu, \nu_{\min 0}$ and, $\nu_{\min 1}$ are used to calculate $\chi_{\text {seis }}^{2}$. The $P_{\mathrm{i}, \text { obs }}$ is non-asteroseismic observed data $\left(T_{\text {eff }}, \log g\right) . N_{\mathrm{s}}$ total number of the data included and $P_{\mathrm{i}, \bmod }$ are non-asteroseismic parameters of the models.

Monte Carlo simulations are used in the error calculations of $M, R, \log g, L$, and $t$ values of the eight evolved stars determined by the model. The uncertainties are listed in Table 2 . The uncertainty of $T_{\bmod }$ is considered the same as $\Delta T_{\mathrm{es}}$. $\Delta Y_{\text {mod }} / Y_{\bmod } \approx 3 \Delta M_{\bmod } / M_{\bmod }$ is obtained. This expression is derived from the interior model data. For the stars $\Delta Z_{\bmod }$ is computed from the observed uncertainty of $[\mathrm{Fe} / \mathrm{H}]$. The mean $\Delta Z_{\text {mod }}$ is determined by averaging all the calculated $\Delta Z_{\text {mod }}$ values. The values of these approaches are determined 
as $\mathrm{Y}=0.2745 \pm 0.0824$ and $\mathrm{Z}=0.0126 \pm 0.0003$ with certain error margins.

\subsection{Mass and radius calculation from scaling relations}

In this study, stellar $M$ and $R$ values are calculated based on three different scaling relations: relations are conventional scaling relations, modified scaling relations, and the Bellinger method (Bellinger 2019). However, conventional and modified scaling relations methods were derived for MS stars. That's why the methods should be tested for evolved stars. The conventional relations are (Kjeldsen \& Bedding 1995):

$\frac{M_{\text {sca }}}{M_{\odot}}=\left(\frac{\nu_{\max }}{\nu_{\max \odot}}\right)^{3}\left(\frac{\Delta \nu}{\Delta \nu_{\odot}}\right)^{-4}\left(\frac{T_{\text {eff }}}{T_{\text {eff } \odot}}\right)^{1.5}$,

and

$\frac{R_{\mathrm{sca}}}{R_{\odot}}=\left(\frac{\nu_{\max }}{\nu_{\max \odot}}\right)\left(\frac{\Delta \nu}{\Delta \nu_{\odot}}\right)^{-2}\left(\frac{T_{\text {eff }}}{T_{\text {eff } \odot}}\right)^{0.5}$.

$\nu_{\max , \odot}$ and $\Delta \nu_{\odot}$ are taken as $\nu_{\max , \odot}=3050 \mu \mathrm{Hz}($ Kjeldsen \& Bedding 1995) and $\Delta \nu_{\odot}=135.15 \mu \mathrm{Hz}$ from BiSON solar data (Chaplin et al. 2014).

Secondly, $M_{\mathrm{III}}$ and $R_{\mathrm{III}}$ are calculated from the modified scaling relation from Paper III. This work assumed that the first adiabatic exponent at the stellar surface $\left(\Gamma_{1 \mathrm{~s}}\right)$ affects the relationship between $\Delta \nu$ and the square root of its mean density and developed a new relation between $\Delta \nu, \rho$, and $\Gamma_{1 \mathrm{~s}}$. The modified scaling relations are as follows:

$\frac{M_{\mathrm{III}}}{M_{\odot}}=\left(\frac{\nu_{\max }}{\nu_{\max \odot}}\right)^{3}\left(\frac{\Delta \nu}{\Delta \nu_{\odot}}\right)^{-4}\left(\frac{T_{\mathrm{eff}}}{T_{\mathrm{eff} \odot}} \frac{\Gamma_{1 \mathrm{~s} \odot}}{\Gamma_{1 \mathrm{~s}}}\right)^{1.5}\left(\frac{f_{\Delta \nu}^{4}}{f_{\nu}^{3}}\right)$

and

$\frac{R_{\mathrm{III}}}{R_{\odot}}=\left(\frac{\nu_{\max }}{\nu_{\max \odot}}\right)\left(\frac{\Delta \nu}{\Delta \nu_{\odot}}\right)^{-2}\left(\frac{T_{\mathrm{eff}}}{T_{\text {eff } \odot}} \frac{\Gamma_{1 \mathrm{~s} \odot}}{\Gamma_{1 \mathrm{~s}}}\right)^{0.5}\left(\frac{f_{\Delta \nu}^{2}}{f_{\nu}}\right)$.

Thirdly, $M_{\text {bel }}$ and $R_{\text {bel }}$ are calculated from Python code derived from the Bellinger method (2019). In this code, the observed $\nu_{\max }, \Delta \nu, T_{\text {eff }}$, and $[\mathrm{Fe} / \mathrm{H}]$ values are entered together with errors. In this way, values of $M, R$, and $t$ are calculated from the scaling relations developed for evolved stars. The $M$ and $R$ of the stars obtained by these relations are given in Table 4.

\subsection{Mass and radius calculation from MIST table}

The $M_{\pi}$ and $R_{\pi}$ are computed using the MIST bolometric correction table (Choi et al. 2016; Dotter 2016). Thus, $L$ values are determined for the stars for whiche the distances are known. From this calculated $L$ values, the $R$ and $M$ values can be determined by the classical method.

While calculating $M_{\pi}$ and $R_{\pi}$ using this method, Gaia DR3 distance and $\mathrm{G}$ magnitude, $T_{\mathrm{es}}$, and logg input parameters are used. The trigonometric parallaxes from Gaia DR3 are known to contain a zero-point offset that has a complex dependence on other observational parameters (e.g. the colour and magnitude of the stars). In this study, the parallax offset is taken 0.028 mas (Huang et al. 2021).

First, the stellar $L$ is calculated for the stars using the MIST bolometric correction table. $\mathrm{R}$ is obtained by the $\mathrm{L}=4 \pi R^{2} \sigma T_{\text {eff }}{ }^{4}$ equation. After determining the radius, the

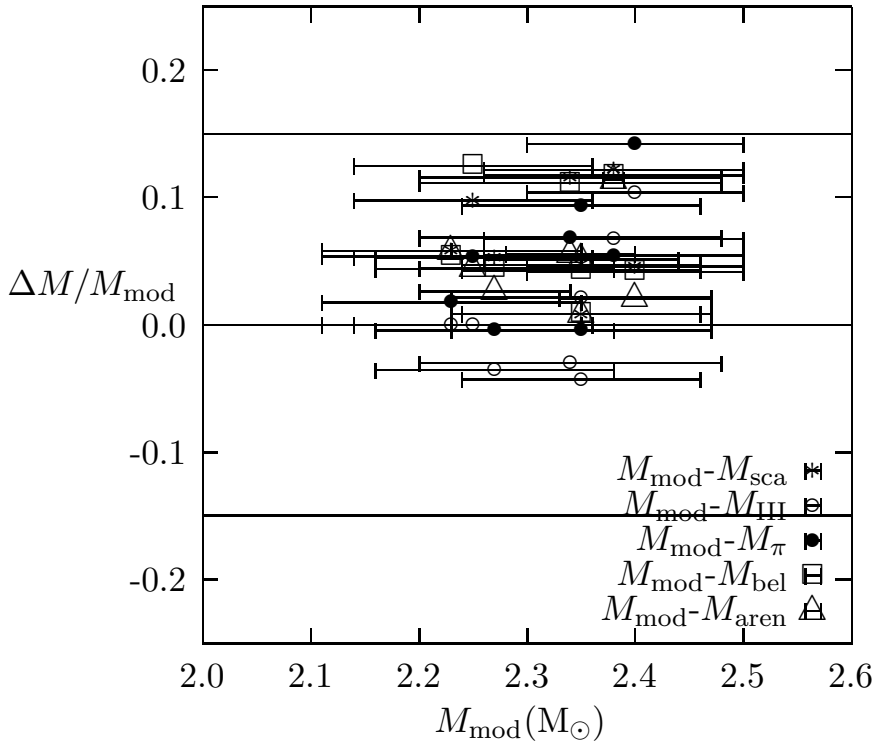

Figure 3. The mass differences are plotted for eight solar-like oscillating evolved star in the NGC 6811. The asterisks, circles, filled circles, squares, and triangles indicate $M_{\bmod }-M_{\text {sca }}, M_{\bmod }-M_{\mathrm{III}}$, $M_{\text {mod }}-M_{\pi}, M_{\text {mod }}-M_{\text {bel }}$, and $M_{\text {mod }}-M_{\text {aren }}$ respectively. The lines for -0.15 and 0.15 .

$M$ is calculated from the $g=G M / R^{2}$ relation. The precision of determining $M$ by this method depends on the sensitivity of the $\log g$ value derived from the observations. For this reason, the observed logg value determined from the asteroseismic data is preferred, when obtaining the $M_{\pi}$ and $R_{\pi}$ of the stars. $M_{\pi}$ and $R_{\pi}$ values calculated by the classical method are listed in Table 4.

\section{RESULTS}

\subsection{Mass and radius comparisons}

Determining the stellar masses by direct observations is difficult, except for eclipsing binary stars. However, stellar $M$ can be determined using observational data with scaling relations developed with asteroseismology. In this study, $M_{\text {sca }}, M_{\text {III }}$ and $M_{\text {bel }}$ are calculated from conventional, modified scaling relations, and Bellinger method, respectively (see Sec. 3.2). In addition, the masses of those stars for which distances are known are calculated by the classical method $\left(M_{\pi}\right)$ (see Sec. 3.3). Lastly, $M_{\text {aren }}$ is taken from Arentoft et al. (2017). The $M_{\text {aren }}$ is obtained using BaSTI grid modeling method. They fit the observed asteroseismic and atmospheric quantities to a grid of BaSTI (Pietrinferni et al. 2004) isochrones using the Bayesian Stellar Algorithm (Silva Aguirre et al. 2015, 2017). The masses calculated using these different methods are compared with those determined from the models $\left(M_{\text {mod }}\right)$. In Fig. 3 , the asterisks, circles, filled circles, squares, and triangles indicate the $M_{\bmod }-M_{\text {sca }}, M_{\bmod }-M_{\mathrm{III}}, M_{\bmod }-M_{\mathrm{bel}}, M_{\bmod }-M_{\pi}$, and $M_{\text {mod }}-M_{\text {aren }}$ respectively. In Fig 3 , in general, the difference between model and calculated $M$ values are less than $1.5 \%$. The model mass of KIC 9655101 is the same as $M_{\mathrm{III}}$. The harmony between the $M_{\bmod }$ and $M_{\pi}$ is shown in Fig. 3. All $M$ values are listed in Table 4 .

The observed oscillation frequencies, particularly $\Delta \nu$, are 
Table 3. Asteroseismic parameters of MESA model results of the eight evolved stars. $\left\langle\Delta \nu_{\mathrm{M}}\right\rangle, \nu_{\max , \mathrm{M}}, \nu_{\min 0, \mathrm{M}}, \nu_{\min 1, \mathrm{M}}$, and $\nu_{\min 2, \mathrm{M}}$ are, respectively, large separation between model oscillation frequencies, model oscillation frequency of maximum amplitude, reference frequencies of model in $\mu \mathrm{Hz}$ units. $\nu_{\max , \mathrm{M}}$ is computed from scaling relations with $T_{\mathrm{eff}, \mathrm{M}}$ and $\log g_{\mathrm{M}} \cdot \chi_{\text {seis }}^{2}$ of the model is in the last column. Typical uncertainties for the reference frequencies are $\left\langle\Delta \nu_{\mathrm{M}}\right\rangle / 2$.

\begin{tabular}{lcrrrrr}
\hline KIC & $\begin{array}{r}\left\langle\Delta \nu_{\mathrm{M}}\right\rangle \\
(\mu \mathrm{Hz})\end{array}$ & $\begin{array}{r}\nu_{\max , \mathrm{M}}(\mu \mathrm{Hz}) \\
(\mu \mathrm{Hz})\end{array}$ & $\begin{array}{r}\nu_{\min , \mathrm{M}} \\
(\mu \mathrm{Hz})\end{array}$ & $\begin{array}{r}\nu_{\min 2, \mathrm{M}} \\
(\mu \mathrm{Hz})\end{array}$ & $\chi_{\text {seis }}^{2}$ \\
\hline 9409513 & 6.02 & 68.21 & 79.86 & 59.04 & 53.42 & 0.69 \\
9532903 & 7.53 & 93.17 & 106.83 & 81.19 & 61.65 & 0.45 \\
9534041 & 8.32 & 114.11 & 83.86 & 62.285 & 61.66 & 0.47 \\
9655101 & 7.88 & 96.55 & 103.45 & 83.74 & 64.22 & 0.32 \\
9655167 & 8.04 & 98.256 & 107.23 & 85.84 & 64.71 & 0.65 \\
9716090 & 8.49 & 116.176 & 119.45 & 92.14 & 69.07 & 0.43 \\
9716522 & 4.87 & 56.159 & 57.53 & 47.45 & 33.74 & 0.45 \\
9776739 & 7.62 & 93.168 & 104.04 & 77.93 & 62.70 & 0.48 \\
\hline
\end{tabular}

Table 4. Listing of all obtained masses and radii of the eight evolved stars. $M_{\text {sca }}$ and $R_{\text {sca }}$ are calculated from conventional scaling relations. $M_{\mathrm{III}}$ and $R_{\mathrm{III}}$ are determined using formulas from Paper III. $M_{\pi}$ and $R_{\pi}$ are calculated from classic method. $M_{\mathrm{bel}}$ and $R_{\mathrm{bel}}$ are calculated from Python code delivered by Bellinger (2019). $M_{\text {aren }}$ and $R_{\text {aren }}$ are taken from Arentoft et al. (2017). MESA masses and radii are given as $M_{\text {mod }}$ and $R_{\text {mod }}$, respectively.

\begin{tabular}{|c|c|c|c|c|c|c|c|c|c|c|c|c|}
\hline $\mathrm{KIC}$ & $\begin{array}{c}M_{\text {sca }} \\
\left(M_{\odot}\right)\end{array}$ & $\begin{array}{c}R_{\text {sca }} \\
\left(R_{\odot}\right)\end{array}$ & $\begin{array}{c}M_{\text {III }} \\
\left(M_{\odot}\right)\end{array}$ & $\begin{array}{c}R_{\mathrm{III}} \\
\left(R_{\odot}\right)\end{array}$ & $\begin{array}{r}M_{\pi} \\
\left(M_{\odot}\right)\end{array}$ & $\begin{array}{r}R_{\pi} \\
\left(R_{\odot}\right)\end{array}$ & $\begin{array}{c}M_{\text {bel }} \\
\left(M_{\odot}\right)\end{array}$ & $\begin{array}{c}R_{\text {bel }} \\
\left(R_{\odot}\right)\end{array}$ & $\begin{array}{l}M_{\text {aren }} \\
\left(M_{\odot}\right)\end{array}$ & $\begin{array}{l}R_{\text {aren }} \\
\left(R_{\odot}\right)\end{array}$ & $\begin{array}{r}M_{\bmod } \\
M_{\odot}\end{array}$ & $\begin{array}{r}R_{\text {mod }} \\
R_{\odot}\end{array}$ \\
\hline 9409513 & $2.29 \pm 0.12$ & $10.46 \pm 0.20$ & $2.15 \pm 0.05$ & $9.69 \pm 0.07$ & $2.06 \pm 0.09$ & $9.92 \pm 0.10$ & $2.30 \pm 0.13$ & $10.58 \pm 0.21$ & $2.35 \pm 0.07$ & $10.61 \pm 0.13$ & $2.40 \pm 0.10$ & $10.69 \pm 0.09$ \\
\hline 9532903 & $2.15 \pm 0.12$ & $8.83 \pm 0.17$ & $2.35 \pm 0.07$ & $9.08 \pm 0.07$ & $2.28 \pm 0.07$ & $9.05 \pm 0.07$ & $2.17 \pm 0.13$ & $8.94 \pm 0.20$ & $2.21 \pm 0.07$ & $9.00 \pm 0.13$ & $2.27 \pm 0.11$ & $8.92 \pm 0.08$ \\
\hline 9534041 & $2.09 \pm 0.10$ & $8.17 \pm 0.13$ & $2.22 \pm 0.05$ & $8.81 \pm 0.04$ & $2.25 \pm 0.05$ & $8.46 \pm 0.09$ & $2.10 \pm 0.10$ & $8.26 \pm 0.13$ & $2.11 \pm 0.01$ & $8.24 \pm 0.06$ & $2.38 \pm 0.12$ & $9.05 \pm 0.08$ \\
\hline 9655101 & $2.24 \pm 0.11$ & $8.70 \pm 0.14$ & $2.45 \pm 0.03$ & $8.97 \pm 0.03$ & $2.11 \pm 0.03$ & $8.41 \pm 0.03$ & $2.25 \pm 0.10$ & $8.79 \pm 0.15$ & $2.23 \pm 0.09$ & $8.75 \pm 0.70$ & $2.35 \pm 0.11$ & $9.09 \pm 0.09$ \\
\hline 9655167 & $2.03 \pm 0.15$ & $8.29 \pm 0.20$ & $2.25 \pm 0.03$ & $8.57 \pm 0.03$ & $2.13 \pm 0.03$ & $8.41 \pm 0.03$ & $1.97 \pm 0.14$ & $8.25 \pm 0.20$ & $2.15 \pm 0.05$ & $8.49 \pm 0.10$ & $2.25 \pm 0.11$ & $8.79 \pm 0.09$ \\
\hline 9716090 & $2.10 \pm 0.13$ & $8.17 \pm 0.13$ & $2.23 \pm 0.03$ & $8.35 \pm 0.03$ & $2.19 \pm 0.03$ & $8.58 \pm 0.03$ & $2.11 \pm 0.11$ & $8.15 \pm 0.15$ & $2.10 \pm 0.00$ & $8.07 \pm 0.00$ & $2.23 \pm 0.12$ & $8.47 \pm 0.08$ \\
\hline 9716522 & $2.33 \pm 0.17$ & $12.12 \pm 0.30$ & $2.30 \pm 0.05$ & $11.87 \pm 0.02$ & $2.36 \pm 0.05$ & $12.20 \pm 0.05$ & $2.33 \pm 0.15$ & $12.24 \pm 0.28$ & $2.33 \pm 0.11$ & $12.21 \pm 0.16$ & $2.35 \pm 0.12$ & $12.38 \pm 0.12$ \\
\hline 9776739 & $2.07 \pm 0.15$ & $8.64 \pm 0.21$ & $2.41 \pm 0.05$ & $9.14 \pm 0.05$ & $2.18 \pm 0.05$ & $8.77 \pm 0.05$ & $2.08 \pm 0.10$ & $8.73 \pm 0.16$ & $2.21 \pm 0.06$ & $8.91 \pm 0.16$ & $2.34 \pm 0.14$ & $9.24 \pm 0.09$ \\
\hline
\end{tabular}

related on the stellar $R$, because the observed $\Delta \nu$ in a star depends on the mean density and the mean density is inversely proportional to the radius. If the observed oscillation frequencies are fitted from the model oscillation frequencies, the stellar $R$ is determined very precisely from interior models. In Fig. 4, the radii obtained from different asteroseismic methods and the classic methods were compared to the radius obtained from the model. The stellar $R$ values calculated from different methods are in good agreement with $R_{\text {mod. }} R$ values are listed in Table 4 . The difference between the $R_{\bmod }$ and $R_{\mathrm{sca}}, R_{\mathrm{III}}, R_{\mathrm{bel}}$, and $R_{\text {aren }}$ is $1.0 \%$. In addition, the difference between $R_{\pi}$ and $R_{\bmod }$ is about less than $1.0 \%$.

\subsection{Reference frequency comparison}

The minima obtained from the model and the observed oscillation frequencies are compared for $l=0$ because other $l$ values are included mixed modes. In Fig. 2 , the $\Delta \nu$ versus $\nu$ graph of KIC 9776739 shows a harmony between the observed and model minimum oscillation frequencies. As seen in the pattern formed by observed and model oscillation frequencies, are quite compatible with each other.

Fig. 5 plots the model and observed $\nu_{\min 0}$ and $\nu_{\min 1}$ of each star in terms of plots of $\Delta \nu$. Fig. 5 compares the values of observed and model $\nu_{\min 0}$ and $\nu_{\min 1}$. The asterisks and filled circles are represented values of $\nu_{\min 0}$ determined by model and observed oscillation frequencies, respectively. The filled circles and squares are represented values of $\nu_{\min 1}$ obtained from observed and model oscillation frequencies, respectively. The figure clearly shows that the observed and model $\nu_{\min 0}$

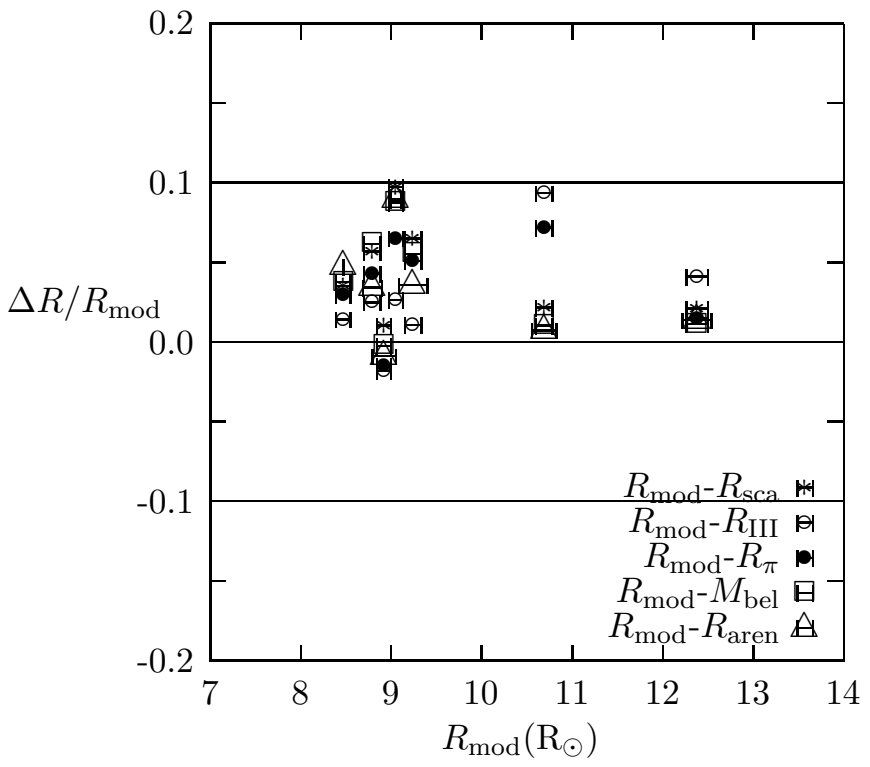

Figure 4. The stellar radii differences are plotted for eight solar-like oscillating stars in the NGC 6811 . The asterisks, circles, filled circles, squares, and triangles indicate $R_{\text {mod }}-R_{\text {sca }}, R_{\text {mod }}-R_{\text {III }}, R_{\text {mod }}-$ $R_{\pi}, R_{\mathrm{mod}}-R_{\text {bel }}$, and $R_{\text {mod }}-R_{\text {aren }}$ respectively. The lines are for -0.10 and 0.10 .

and $\nu_{\min 1}$ are in excellent agreement. This indicates that the observed and model reference frequencies are just as congruent as the observed and model values of $\Delta \nu$. The $\nu_{\min 0}$ and $\nu_{\min 1}$ values are listed in Table 3 . 


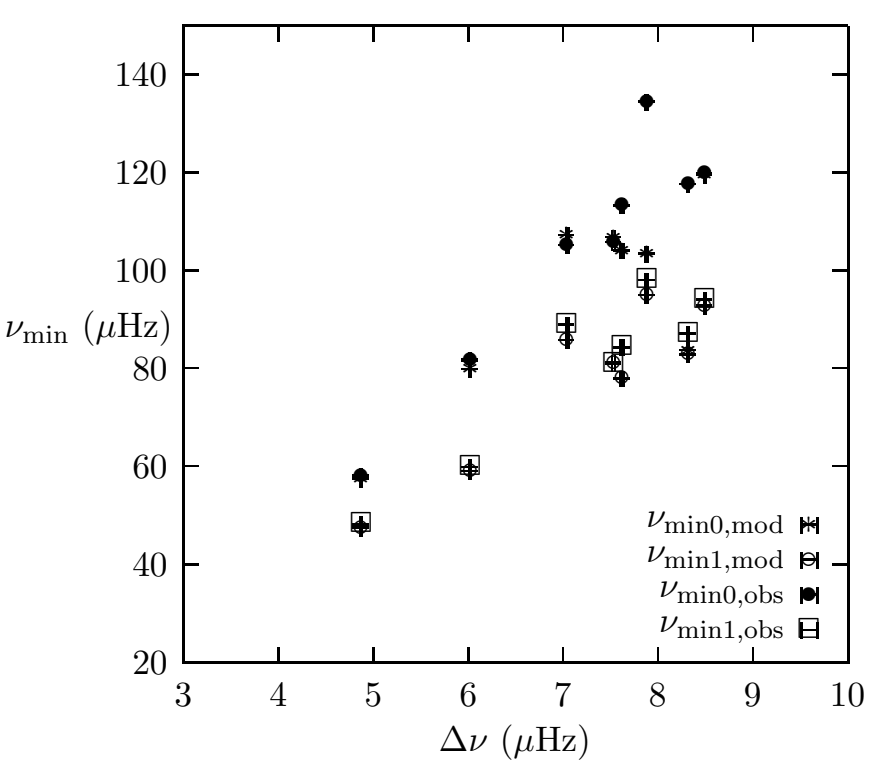

Figure 5. Plot of $\Delta \nu$ as a function of the reference frequencies $\left(\nu_{\min 0}\right.$ and $\left.\nu_{\min 1}\right)$ for the stars. The asterisks and filled circles are the represented values of $\nu_{\text {mino }}$ obtained from model and observed oscillation frequencies, respectively. The circle and square are represented values of $\nu_{\min 1}$ obtained from observed and model oscillation frequencies, respectively.

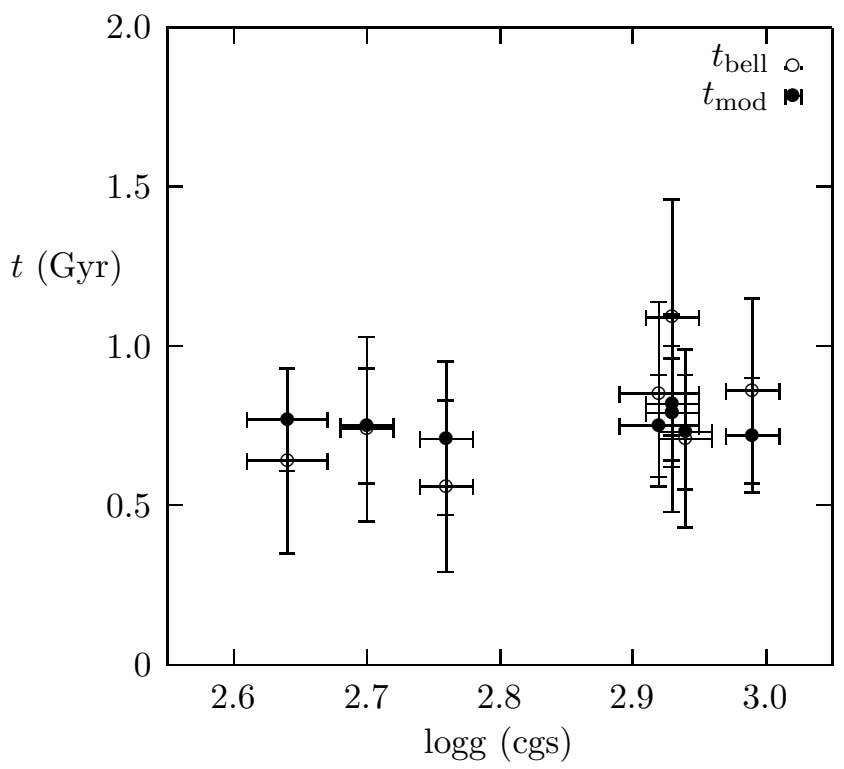

Figure 6. The plot of stellar ages as function of the logg for the stars. The circles and filled circles are represented values of stellar age obtained from Bellinger (2019) and interior models, respectively.

In addition, the $\chi_{\text {seis }}^{2}$ values are calculated from Eq. 5 . These values show that the model calculations for the stars are highly compatible with the observations. The model and the observed oscillation frequencies in terms of $\nu_{\min 0}, \nu_{\min 1}$, $\nu_{\max }$, and $\Delta \nu$ are used for calculated $\chi_{\text {seis }}^{2}$. The calculated $\chi_{\text {seis }}^{2}$ are listed in Table 3 .
Table 5. The ages of the NGC 6811 obtained by different methods. In the table, age and errors, and reference information are given, respectively.

\begin{tabular}{lc}
\hline Age & ref \\
$(\mathrm{Gyr})$ & \\
& \\
\hline $1.05 \pm 0.05$ & Sandquist et al. 2016 \\
$1.04 \pm 0.07$ & Curtis et al. 2019 \\
$1.00 \pm 0.02$ & Spada \& Lanzafame 2020 \\
$1.00 \pm 0.07$ & Cargile et al. 2014 \\
$1.00 \pm 0.02$ & Meibom et al. 2011 \\
$1.00 \pm 0.04$ & Rodriguez et al. 2020 \\
$0.94 \pm 0.08$ & Janes et al. 2013 \\
$0.87 \pm 0.02$ & Bossini et al. 2019 \\
$0.70 \pm 0.10$ & Glushkova et al. 1999 \\
$0.64 \pm 0.02$ & Donor et al. 2020 \\
\hline
\end{tabular}

\subsection{Age comparison}

Determining the stellar ages is critical for improve our understanding of stellar structure and evolution. However, it is quite difficult to determine the stellar ages by observations. Nevertheless, the age of a star is determined by interior models. In this study, the stellar age is determined by constructed models using asteroseismic constraints. Moreover, the solarlike oscillating stars studied are members of the open cluster. Thus, the range of model ages is $0.71-0.82$ Gyr. The model stellar ages are listed in Table 2 .

The ages of NGC 6811 reported in different studies are listed in Table 5. In Table 5, the oldest $t$ is 1.05 Gyr (Sandquist et al. 2016), while the youngest $t$ is $0.64 \mathrm{Gyr}$ (Donor et al. 2020). A reason accounting for these age differences could be the methods used. For example, Meibom et al. (2011) examined the NGC 6811 cluster in detail, along with the relationship between the stellar rotation periods and their masses and ages. Thus, the age of this cluster was determined to be approximately 1 Gyr. Another reason accounting for the difference may be model input chemical composition. For example, 36 Kepler short-cadence stars in NGC 6811 were anaysed by Rodriguez et al. (2020). Their star types include two solar-like oscillating red giant stars, $21 \mathrm{MS}$ pulsators (16 $\delta$ Scuti and five $\gamma$ Doradus stars), and 13 rotating variables. They found that the age of cluster is $1 \mathrm{Gyr}$ from Padova stellar isochrone for $\mathrm{Z}=0.012$. On the other hand, NGC 6811 cluster is characterized as a young (0.7 $\pm 0.1 \mathrm{Gyr})$ and possibly solar-metallicity star population by Glushkova et al. (1999). In this study, the models constructed with the MESA evolution code are taken as $Z=0.0126$ and average age is approximately 0.76 Gyr.

Finally, the stellar ages are computed using the Python code developed by Bellinger (2019). With this code, the ages of evolved stars are obtained by using observational asteroseismic parameters without constructed interior model. The ages obtained from the model and Bellinger's method are compared in Fig. 6. The $t$ range calculated from Bellinger is $0.56-0.74$ Gyr. This age range is very compatible with the age range obtained by a model constructed in this study. 
When the $t$ of the literature and the cluster members in this study are compared, the minimum difference is $0.01 \pm 0.28$ Gyr. This calculated value is still within the error range of the model ages. Thus, it can be concluded that the different stellar ages obtained for these cluster members are quite compatible with the model ages.

\section{CONCLUSIONS}

This study analyses eight solar-like oscillating evolved stars which are NGC 6811 cluster members. These selected stars are modelled for the first time in this study. The stellar models have been constructed by using MESA code. The interior models of the stars are included the mass-loss method. The oscillation frequencies of eight evolved stars are examined. The mean large separation, frequencies of the maximum amplitude, and reference frequencies are computed by stellar models.

The fundamental stellar properties are determined in the interior models using these asteroseismic parameters. The observed asteroseismic $\left(\Delta \nu, \nu_{\max }, \nu_{\min 0}\right.$, and $\left.\nu_{\min 1}\right)$, and nonasteroseismic ( $T_{\text {eff }}, \log g$, and $\left.[\mathrm{Fe} / \mathrm{H}]\right)$ parameters are compared with model values, which are obtained from MESA evolution code for the stars. The compatibility between the model and observed oscillation frequencies was examined. For this, reference frequencies and $\Delta \nu$ obtained from the model and observation are compared. It was observed that values of the model $\nu_{\min 1}$ and $\nu_{\min 0}$ are compatible with the observational $\nu_{\min 1}$ and $\nu_{\min 0}$ frequencies. Moreover, attention was paid to ensure that the model $\Delta \nu$ value is very close the observational value. These methods allow us to determine the fundamental stellar parameters to high precision. The stellar models are constructed using mass-loss methods $(\eta=0.1)$. The total mass loss in all the interior models constructed is 0.01 $M_{\odot}$. The stars have masses and radii spanning of $2.23-2.40$ $M_{\odot}$ and $8.47-12.38 R_{\odot}$, respectively, with typical uncertainties of $\sim 11$ per cent in mass and $\sim 9$ per cent in radius.

Furthermore, the conventional, modified scaling relations, and the classical method have been used to calculated the masses and radii of the evolved stars. The masses and radii obtained from the different methods are compared with those derived by the model. The radii obtained from the model and those obtained from different methods are close to each other. However, the stellar mass determined by scaling relations, and the model mass is slightly different from each other. In addition, the agreement between the model masses and those obtained from the classical method is very good at such stars.

After stellar mass, chemical composition is the most important parameter that has an impact on the stellar structure and evolution. However, determining the metal abundance of a star is very difficult. Studying cluster member stars with the same metal abundance provides an advantage in this regard. The method widely reported in the literature is the calculation of the metal abundance of stars from $[\mathrm{Fe} / \mathrm{H}]$ or $[\mathrm{M} / \mathrm{H}]$. In this study, it is calculated by averaging the calculated $Z$ values of the stars that are members of the NGC 6811 cluster. The stellar metallicity is calculated from mean metallicity and $Z$ is set to $0.0126 \pm 0.0003$.

In this study, the stellar age is calculated from MESA models for the stars. Model stellar ages are listed in Table 2. The stellar model age range is 0.71-0.82 Gyr. In addition, the age determined using different methods was compared with the model ages. When comparing the ages obtained from these different methods with MESA models, the minimum difference is $0.01 \pm 0.28 \mathrm{Gyr}$. Also, the $t$ range calculated from the Python code developed by Bellinger (2019) is 0.56-0.74 Gyr. The stellar $t$ obtained from the models is compatible with the literature. The fact that the age values calculated by different methods are close to each other indicates how well the model ages are obtained with the methods applied in this study.

\section{ACKNOWLEDGEMENTS}

I would like to thank Dr. Mutlu Yıldız for sharing his invaluable experiences and contributing in this study with Gaia database analysis, and Sibel Örtel, who has been a great help in modeling process. I am grateful to Ege University Planning and Monitoring Coordination of Organizational Development and Directorate of Library and Documentation for their support in editing and proofreading service of this study. I would also like to thank my son Emirhan Sami and my husband Mustafa Orhan for their support. This work is supported by the Scientific and Technological Research Council of Turkey (TÜBITAK:118F352). I would like to thank Kelly Spencer for her kind help in checking the language of the revised manuscript.

\section{DATA AVAILABILITY}

The data underlying this article will be shared on reasonable request to the corresponding author.

\section{REFERENCES}

Angelou G. C., Bellinger E. P., Hekker S., Mints A., Elsworth Y., Basu S., Weiss A., 2020, MNRAS, 493, 4987

Angulo C. et al., 1999, Nucl. Phys. A, 656, 3

Arentoft T., Brogaard K., Jessen-Hansen J., Silva Aguirre V., Kjeldsen H., Mosumgaard J. R., Sandquist E. L., 2017, ApJ, 838, 115. doi:10.3847/1538-4357/aa63f7

Basu S., Grundahl F., Stello D., Kallinger T., Hekker S., Mosser B., García R. A., et al., 2011, ApJL, 729, L10. doi:10.1088/20418205/729/1/L10

Asplund M., Grevesse N., Sauval A. J., Scott P., 2009, ARA\&A, 47, 481. doi:10.1146/annurev.astro.46.060407.145222

Bedding T. R., Mosser B., Huber D., Montalbán J., Beck P., Christensen-Dalsgaard J., Elsworth Y. P., et al., 2011, Natur, 471, 608. doi:10.1038/nature09935

Bossini D., Vallenari A., Bragaglia A., Cantat-Gaudin T., Sordo R., Balaguer-Núñez L., Jordi C., et al., 2019, A\&A, 623, A108. doi:10.1051/0004-6361/201834693

Böhm- Vitense E., 1958, Z. Astrophys., 46, 108

Caughlan G. R., Fowler W. A., 1988, At. Data Nucl. Data Tables, 40, 283

Cargile P. A., James D. J., Pepper J., Kuhn R. B., Siverd R., Stassun K. G., 2014, ApJ, 782, 29. doi:10.1088/0004$637 \mathrm{X} / 782 / 1 / 29$

Çelik Orhan Z., Yıldız M., Kayhan C., 2021, MNRAS, 503, 4529. doi:10.1093/mnras/stab757

Chaplin W. J. et al., 2014, ApJS, 210, 1

Christensen-Dalsgaard J., 2008, Ap\&SS, 316, 113

Choi J., Dotter A., Conroy C., Cantiello M., Paxton B., Johnson B. D., 2016, ApJ, 823, 102. doi:10.3847/0004-637X/823/2/102 
Curtis J. L., Agüeros M. A., Douglas S. T., Meibom S., 2019, ApJ, 879, 49. doi:10.3847/1538-4357/ab2393

Donor J., Frinchaboy P. M., Cunha K., O'Connell J. E., Allende Prieto C., Almeida A., Anders F., et al., 2020, AJ, 159, 199. doi:10.3847/1538-3881/ab77bc

Dotter A., 2016, ApJS, 222, 8. doi:10.3847/0067-0049/222/1/8

Bellinger E. P., 2019, MNRAS, 486, 4612

Edvardsson, B., Andersen, J., Gustafsson, B, et al., 1993, $\mathrm{A} \& \mathrm{~A}, 275,101 \mathrm{E}$

Ferguson J. W., Alexander D. R., Allard F., Barman T., Bodnarik J. G., Hauschildt P. h., Heffner- Wong A., Tammanai A., 2005, ApJ, 623, 585

Glushkova E. V., Batyrshinova V. M., Ibragimov M. A., 1999, AstL, 25, 86

Guggenberger E., Hekker S., Basu S., Bellinger E., 2016, MNRAS, 460,4277

Hawkins K., Masseron T., Jofré P., Gilmore G., Elsworth Y., Hekker S., 2016, A\&A, 594, A43. doi:10.1051/0004$6361 / 201628812$

Hekker S., Basu S., Stello D., Kallinger T., Grundahl F., Mathur S., García R. A., et al., 2011, A\&A, 530, A100. doi:10.1051/0004$6361 / 201016303$

Huang Y., Yuan H., Beers T. C., Zhang H., 2021, ApJL, 910, L5. doi:10.3847/2041-8213/abe69a

Iglesias C. A., Rogers F. J., 1993, ApJ, 412, 752

Iglesias C. A., Rogers F. J., 1996, ApJ, 464, 943

Janes K., Barnes S. A., Meibom S., Hoq S., 2013, AJ, 145, 7. doi:10.1088/0004-6256/145/1/7

Kjeldsen H., Bedding T. R., 1995, A\&A, 293, 87

Meibom S., Barnes S. A., Latham D. W., Batalha N., Borucki W. J., Koch D. G., Basri G., et al., 2011, ApJL, 733, L9. doi:10.1088/2041-8205/733/1/L9

Miglio A., Brogaard K., Stello D., Chaplin W. J., D'Antona F., Montalbán J., Basu S., et al., 2012, MNRAS, 419, 2077. doi:10.1111/j.1365-2966.2011.19859.x

Mosser B. et al., 2013, A\&A, 559, A137

Paxton B., Bilsten L., Dotter A., Herwing F., Lesaffre P., Timmes F., 2011, ApJS, 2011, 192

Paxton B., Catiello M., Arras P., Bildsten L., Brown E. F., Dotter A., Mankovich C., Montgomery M. H. et al., 2013, ApJS, 208

Reimers D., 1975, MSRSL, 8, 369

Renzini A., Fusi Pecci F., 1988, ARA\&A, 26, 199. doi:10.1146/annurev.aa.26.090188.001215

Rodríguez E., Balona L. A., López-González M. J., Ocando S., Martín-Ruiz S., Rodríguez-López C., 2020, MNRAS, 491, 4345. doi:10.1093/mnras/stz3143

Sandquist E. L., Jessen-Hansen J., Shetrone M. D., Brogaard K., Meibom S., Leitner M., Stello D., et al., 2016, ApJ, 831, 11. doi:10.3847/0004-637X/831/1/11

Sharma S., Stello D., Bland-Hawthorn J., Huber D., Bedding T. R., 2016, ApJ, 822, 15

Spada F., Lanzafame A. C., 2020, A\&A, 636, A76. doi:10.1051/0004-6361/201936384

Stello D., Basu S., Bedding T. R., Brogaard K., Bruntt H., Chaplin W.J̃., Christensen-Dalsgaard J., et al., 2010, AN, 331, 985. doi:10.1002/asna.201011442

Stello D., Meibom S., Gilliland R. L., Grundahl F., Hekker S., Mosser B., Kallinger T., et al., 2011a, ApJ, 739, 13. doi:10.1088/0004-637X/739/1/13

Stello D., Huber D., Kallinger T., Basu S., Mosser B. , Hekker S., Mathur S., et al., 2011b, ApJL, 737, L10. doi:10.1088/2041$8205 / 737 / 1 /$ L10

White T. R. et al., 2011, ApJ, 742, L3

Verma K., Faria J. P., Antia H. M., Basu S., Mazumdar A., Monteiro M. J. P. F. G., Appourchaux T., et al., 2014, ApJ, 790, 138. doi:10.1088/0004-637X/790/2

Verma K., Raodeo K., Antia H. M., Mazumdar A., Basu S., Lund M. N., Silva Aguirre V., 2017, ApJ, 837, 47. doi:10.3847/1538-

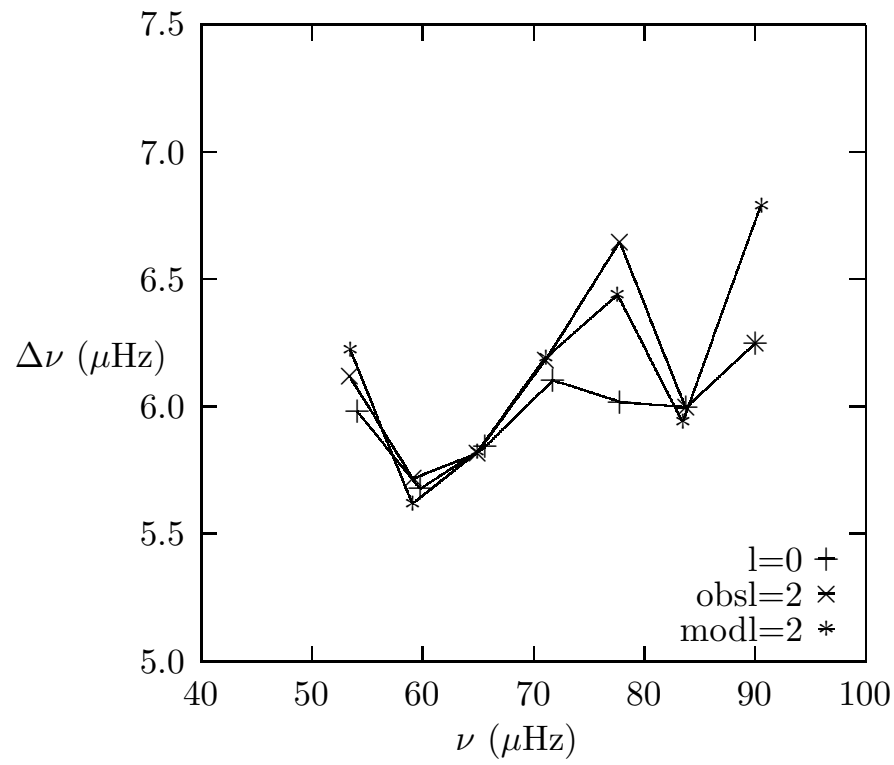

Figure A1. Plot of $\Delta \nu$ as a function of $\nu$ graph for $l=2$ model (MESA, asteriks) and observed $l=0$ and 2 oscillation frequencies (pluses and crosses, respectively) of KIC 9409513.

$4357 /$ aa5da7

Yıldız M., Çelik Orhan Z., Aksoy C., Ok S., 2014a, MNRAS, 441, 2148 (Paper I)

Yıldız M., Çelik Orhan Z., Kayhan C., Turkoglu G. E., 2014b, MNRAS, 445, 4395

Yıldız M., Çelik Orhan Z., Kayhan C., 2015, MNRAS, 448, 3689 (Paper II)

Yıldız M., Çelik Orhan Z., Kayhan C., 2016, MNRAS, 462, 1577 (Paper III)

4277

\section{APPENDIX A: SOME EXTRA MATERIAL}

If you want to present additional material which would interrupt the flow of the main paper, it can be placed in an Appendix which appears after the list of references.

This paper has been typeset from a $\mathrm{TE}_{\mathrm{E}} \mathrm{X} / \mathrm{LT}_{\mathrm{E}} \mathrm{X}$ file prepared by the author. 